\title{
Zur Gliederung der mittelpleistozänen Ablagerungen in Oberschwaben
}

\author{
Von Hans Gra u l. Mit 4 Abb.
}

Auf der Tagung der Deutschen Quartärvereinigung in München 1950 habe jch kurz über dieses Thema referiert, nachdem auf der dreitägigen Vorlandexkursion jener Tagung schon einiges dazu gezeigt worden war (vgl. Graul, Weidenbach \& Schaefer 1951). Den Exkursionsteilnehmern wurde außerdem von der Geologischen Abteilung in Stuttgart ein Probedruck eines neuen geologischen Kartenwerkes 1 : 100000 von Oberschwaben (Blatt Ulm) ausgehändigt. Darin hat der Bearbeiter des Blattes, F. WeIDENBach, als Ergebnis einer jahrelangen kameradschaftlichen Beratung meiner stratigraphischen Arbeiten im Quartär Oberschwabens die Grundgliederung der rißzeitlichen Ablagerungen in drei Gruppen (Alt-, Mittel- und Jungriß) übernommen.

Im Folgenden wird die Dreigliederung der Rißzeit aus praktischen Gründen beibehalten. Es hat sich nämlich im Gebiet des alten Rheingletschers herausgestellt, daß sowohl im Bereich der Altmoränen als auch in dem der Terrassenschotter am ehesten mit drei stratigraphischen Gruppen glazialer Ablagerungen und Formen zu rechnen ist. Diese drei Gruppen, welche weder gleichen Zeitabständen noch je einem einzigen Gletschervorstoß oder -stand entsprechen, unterscheiden sich untereinander 1.) stratigraphisch (durch Schichtdiskordanzen und verschieden mächtige Bedeckung mit periglaziären Bildungen), 2.) geröllpetrographisch (vor allem durch wechselnden Kristallingehalt in ein und derselben Abflußrinne, ferner durch wechselnden Geröllanteil aus dem Schwarzwald und dem süddeutschen Schichtstufenland in der Donaurinne) und 3.) schließlich morphologisch (durch den Grad der nachträglichen Abtragung, Zerschneidung und Verwitterung, wie durch Formendiskordanzen). Diese Unterschiede sind im Grunde die gleichen, die schon seinerzeit für A. PENcK entscheidend waren für die Annahme einer viermaligen Vereisung der Alpen und ihrer Vorländer. Wie sich zeigen wird, hat sich im Gebiet des alten Rheingletschers an der Hauptgruppierung, wie sie F. WeIDENBACH bei seiner Kartierung (1937) gegenüber A. PENCK (1901-09) und M. BRÄUнӓUSER (1921) vorgenommen hat, nichts geändert. Diese Hauptgruppierung F. WeIDEnBach's läßt sich in einem schematischen Profil folgendermaßen darstellen:

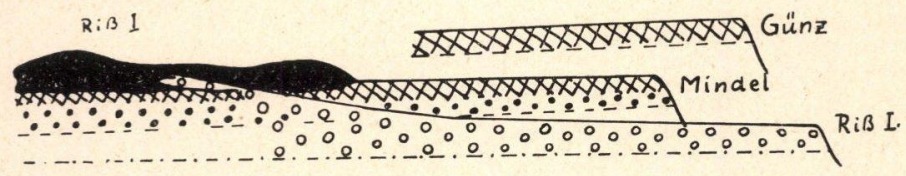

Abb. 1. Schematisches Profil der Schichtlagerung zwischen Riß und Iller (nach WeIDENBACH 1937). Schwarz: Riß 1 = Moräne; Ringel: Riß I = Schotter; Punkte: Mindelschotter; gekreuzt: verwitterte Deckenschotter.

Dagegen hat sich einiges an der Gliederung aller jener Ablagerungen geändert, die WeIDENBAch als ri $B$ zeitlich bezeichnet und die er in zwei Untergruppen, nämlich Riß I und II, geteilt hatte (ähnlich auch bei I. Schaefer 1940, A. SchreINER 1950 und K. SCHÄDEL 1950).

Im Folgenden sollen die mittelpleistozänen Schichtfolgen aus den drei wichtigsten Schmelzwasserrinnen der nördlichen Rheingletscher-Abdachung, aus Donau-, Riß- und Rottal, bereits etwas generalisiert, beschrieben werden. Wenn 
hier auch mehr als drei glaziale Akkumulationen festgestellt werden konnten, so sagt dies noch nichts Endgültiges über die „qualitative“ Bedeutung jeder dieser glazialen Akkumulationen aus, das heißt also, es sagt vor allem nichts darüber aus, ob sie im einzelnen Falle einem Glazial oder nur einem Stadial entsprechen. Wenn auch die stratigraphische und die Formen-Diskordanz fast in jedem Falle zwischen zwei aufeinanderfolgenden Glazialablagerungen einen stärkeren Eisrückzug wahrscheinlich machen, so ist wegen der großmorphologischen Verhältnisse des nördlichen Rheingletschergebietes doch vor voreiligen Schlüssen zu warnen. Denn jede Schwankung des Eises mit einem Zwischenrückzug über die sogenannte Europäische Wasserscheide (Rhein-Donau) nach S, kann sich bei einem neuerlichen Vormarsch des Eises über diese Scheide hinweg ganz ähnlich auswirken wie ein echter Eisrückzug bis in die Alpen mit längerem warmklimatischem Intervall (Interglazial). Es muß daher noch mehr Beweismaterial zur Bestimmung der qualitativen Bedeutung der vorhandenen geologischen und morphologischen Diskordanzen gefunden werden. Manches kann darüber schon im Folgenden gesagt werden. Wenn F. WeIDENBACH in der Farbenerklärung seiner neuen Karte von drei Riß - E is z e i t e n spricht, und C. Troll (1951) ihm in dieser Ansicht folgt, dürfte damit nach meinen bisherigen Geländebeobachtungen kaum zu weit gegangen sein.

\section{Zur Stratigraphie der pleistozänen Ablagerungen im Donautal zwischen Mengen und Ulm}

Die Ausgangsbasis für die Quartärstratigraphie ist auch in Oberschwaben das Donautal, so weit es die Sammelrinne des Alpenvorlandes darstellt. Diese Ausgangsbasis erhält durch die Tatsache noch mehr Gewicht, daß der Gletscher gerade während des Mittelpleistozäns mehrmals das Vorland bis zur Alb erfüllt hat und im Donautal sogar ein ganz ansehnliches Teilzungenbecken, das von Riedlingen, gebildet hat. Es sind hier, mit den jüngsten beginnend, folgende Schichtglieder festzustellen:

1.) Der Schotterkörper von Alth ei m bei Riedlingen: die Oberfläche senkt sich von 547 auf $543 \mathrm{~m}$ und liegt im Mittel $14 \mathrm{~m}$ über dem Talboden der Donau. Im Aufschluß am Wasenplatz südlich Altheim liegt der Schotter auf Geschiebemergel, die Schottermächtigkeit beträgt nur $6-8 \mathrm{~m}$. Das $1-2 \mathrm{~m}$ tief verwitterte Geröll ist hier von einer bis 1,5 m mächtigen Lehmdecke überdeckt. Die Schotteranalyse (siehe Tabelle 1, Nr. 1) zeigt einen hohen Anteil an Alb- und Schwarzwaldmaterial. Letzteres findet sich in den postglazialen Donauschottern des Riedlinger Beckens nur vereinzelt. Sein häufiges Vorkommen im Altheimer Schotter weist auf seine kaltklimatische Entstehung. Andererseits tritt das alpine Geröll, besonders das kalkalpine, so stark gegenüber der „donauglazialen“ Mischung zurück, daß der entsprechende Stand des Rheingletschers kaum über den der Würmzeit hinausgegangen sein kann.

Die Akkumulation von Altheim nimmt größere Flächen ein und bildet die eigentliche Jungriß terrasse im Donautal bis Ulm, so vor allem vom Vöhringerhof östl. Riedlingen über Unlingen bis Burgstall, teils auf Fels, teils auf Geschiebemergel, ferner gegenüber Neuburg bei Ober-Marchtal usw. Sie dürfte korrelat sein mit dem Donaustettener Terrassenschotter im Rißtal und setzt sich fort über die Ulmer Stadtterrasse, die Pfuhler Terrasse zur Langenauer Hochterrasse. Donauaufwärts gehört hierher die aus dem gleichen Material wie bei Riedlingen aufgebaute Schotterterrasse von Sigmaringendorf und Scheer (die von M. SchмiDt 1935 als „Donauschotter der abklingenden Rißeiszeit" kartiert worden ist). 


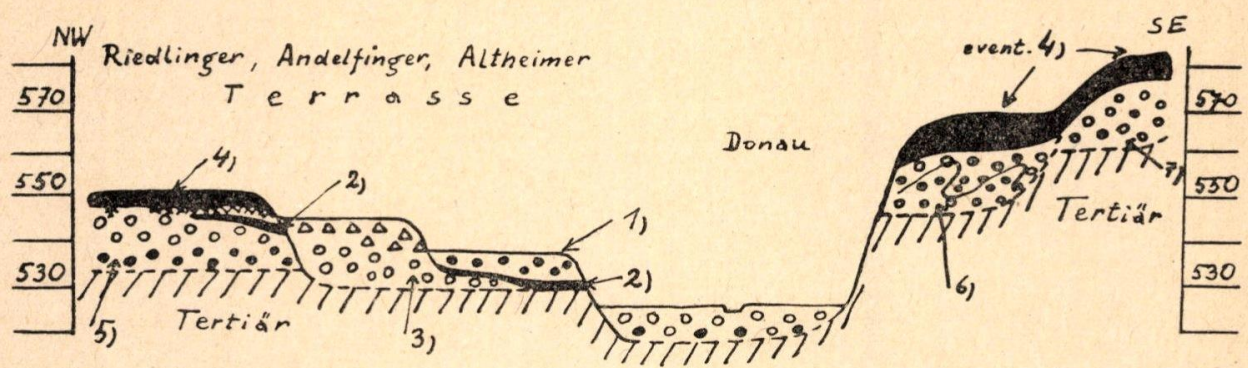

Abb. 2. Schematisches Sammelprofil durch das Riedlinger Becken.

\section{Grundmoräne,}

$\Delta \Delta \Delta$ Schotter, lokale Hangendfazies,

○ $\bigcirc$ ○ Schotter, hochrheinische Fazies,
- Schotter, donauglaziale Fazies, $\times \times \times$ Verwitterung.

Zahlen siehe Schichtfolge im Text.

2.) Der Geschiebemergel im Liegenden von 1.), der außerdem die verschiedensten Schotterkörper überdeckt, von denen die älteren teilweise noch Reste ihrer Verwitterungsdecke erhalten haben. Da dieser Geschiebemergel im Bekken unter den altersmäßig gut zu datierenden Jungrißschottern zu finden ist (so in Altheim und in Unlingen), mußte der Gletscher damals bereits das Becken bis fast zur heutigen Tiefe ausgeräumt haben.

3.) Die zu 2.) gehörenden Vorstoßschotter sind noch unter dem Geschiebemergel bei Unlingen und bei Altheim, an beiden Stellen vom geröllpetrographisch andersartigen Jungrißschotter diskordant überlagert, zu beobachten. Sie sind hier stark vom nachrückenden Gletscher ausgeräumt und, soweit noch erhalten, gestaucht. Ihre Basis ist bei Unlingen ca. $6 \mathrm{~m}$ über dem Donautalboden gelegen. Sie fällt talab etwas flacher ein als der heutige Talboden; so liegt sie im Übergangskegel von Datthausen $8 \mathrm{~m}$, bei Ober-Marchtal $10 \mathrm{~m}$ über Talboden, während sie talauf etwa bei Herbertingen in diesen einstreicht. Die unter dem Übergangskegel von Datthausen durchlaufenden Vorstoßschotter sind über OberMarchtal talab zu verfolgen. Das Material der Herbertinger Kiesgruben zeigt auffallende Stauchungen, die nur von einer nachträglichen Vereisung im Donaubecken stammen können. Der Schotter zeigt genau wie weiter talab eine typische Rheingletschermischung mit einem geringen Hundertsatz aus dem oberen Donautal (siehe Tabelle 1, Nr. 2 und 3). Sie kann als die hochrheinische Geröllfazies bezeichnet werden.

Die Schichten 2.) und 3.) dürften also e i n e m glazialen Komplex angehören und zwar dem weitesten Gletschervorstoß der westlichen und mittleren Teilströme des alten Rheingletschers. Die Ablagerungen dieses Vorstoßes sind im Vorland teilweise noch recht gut erhalten, vor allem aber im alten Donautal durch die $\mathrm{Alb}$, in der sogenannten Kirchener-Schmiech-Blau Talung, denn sie bilden hier die letzte Donaufüllung. Sie überschreiten andererseits aber auch die alte Wasserscheide zwischen Unter-Marchtal und Munderkingen mit einer Basishöhe von $525 \mathrm{~m}$. Ihre Verbreitung läßt erkennen, daß die Verlegung der Donau an den Albrand am Ende jener mächtigen Akkumulation, bedingt durch das besonders weite Talabsteigen des Eises, erfolgt ist. Dieser Vorgang brachte eine Verkürzung des Donaulaufs bis Ulm um über $25 \%$ mit sich, was nicht ohne „einschneidende" Folgen für die neue Donaustrecke blieb. So beobachtet man auch eine relativ starke Ausräumung der bei Marchtal noch über $20 \mathrm{~m}$ mächtigen Aufschotterung des weitesten Vorstoßes und neuerliche Einlagerungen mit einem anderen petrographischen Charakter (ähnlich der Hangendfazies, siehe unter Nr. 4 der Tabelle 1). Entweder gehören diese Bildungen zu größeren Rück- 
zugsstillständen, die meist eine stark lokal gefärbte Materialmischung zeigen (so z. B. der Moränenstand von Heiligkreuztal mit dem Schotterfeld ostwärts von Andelfingen) oder doch noch zum weitesten Stand. Wir hätten dann im neuen Donautal eine Einschneidungszeit während der letzten Phase des Gletschervorrückens. Solche Vorgänge gab es auch bei anderen hochglazialen Talwechseln der Schmelzwässer mit Laufverkürzung.

Tabelle 1

\begin{tabular}{|c|c|c|c|c|c|c|c|c|c|}
\hline Nr. & Ort: & Alter: & KA. & Alb & \multicolumn{3}{|c|}{ Gn. Am. Schw. } & Qu. & Rest. \\
\hline 1 & Altheim & Jungriß & 100 & 170 & \multirow{2}{*}{\multicolumn{2}{|c|}{$\begin{array}{c}113 \\
13^{\prime} 5-18^{\prime} 5\end{array}$}} & 76 & 27 & 84 \\
\hline 2 & $\begin{array}{l}\text { Herbertingen } \\
\text { (3 Proben) }\end{array}$ & Mittelriß & 100 & $6-10$ & & & $0-0$ '4 & 1'7-2'0 & $2{ }^{\prime} 8-7^{\prime} 4$ \\
\hline 3 & Reutlingendorf & $"$ & 100 & 15 & \multicolumn{2}{|c|}{$21^{\prime} 7$} & $1 ' 5$ & 2 & 8 \\
\hline 4 & Datthausen & $"$ & 100 & 124 & \multicolumn{2}{|c|}{$29 ' 5$} & - & $4 ' 8$ & 13 \\
\hline 5 & $\begin{array}{l}\text { Ob. Marchtal } \\
\text { ( } 6 \mathrm{~m} \text { hoch) }\end{array}$ & ” & & $23^{\prime} 6$ & \multicolumn{2}{|c|}{$11^{\prime} 4$} & $0{ }^{\prime} 4$ & $1 ' 1$ & 0 '8 \\
\hline 6 & $\begin{array}{l}\text { Ob. Marchtal } \\
\text { (Basis) }\end{array}$ & ” & 100 & 1890 & \multicolumn{2}{|c|}{$88^{\prime} 0$} & 82 & 22 & 35 \\
\hline 7 & $\begin{array}{l}\text { Riedlingen } \\
6 \mathrm{~m} \text { hoch) }\end{array}$ & $"$ & 100 & $28^{\prime} 3$ & $15^{\prime} 0$ & $6{ }^{\prime} 0$ & $3{ }^{\prime} 0$ & $2^{\prime} 0$ & $11^{\prime} 3$ \\
\hline 8 & $\begin{array}{l}\text { Riedlingen } \\
\text { (11/2 } \mathrm{m} \text { hoch) }\end{array}$ & ” & 100 & 326 & $20 ’ 5$ & $19 ' 5$ & $14^{\prime} 3$ & $10^{\prime} 7$ & $19^{\prime} 0$ \\
\hline 9 & $\begin{array}{l}\text { Ertingen } \\
\text { ( } 7 \mathrm{~m} \text { hoch) }\end{array}$ & Altriß & 100 & $5 ’ 8$ & 20 '1 & $18^{\prime} 3$ & - & $1^{\prime} 0$ & $22{ }^{\prime} 4$ \\
\hline 10 & $\begin{array}{l}\text { Ertingen } \\
\text { (3-4 m hoch) }\end{array}$ & $"$ & 100 & 130 & $61^{\prime} 0$ & $9^{\prime} 0$ & $4 ' 3$ & $13 ’ 2$ & $33{ }^{\prime} 0$ \\
\hline 11 & $\begin{array}{l}\text { Ertingen } \\
\text { ( } 2 \mathrm{~m} \text { hoch) }\end{array}$ & ” & 100 & $2{ }^{\prime} 0$ & $16^{\prime} 6$ & $3 ’ 0$ & - & $3{ }^{\prime} 4$ & $6^{\prime} 2$ \\
\hline 12 & $\begin{array}{l}\text { Baustetten } \\
\text { (2 Proben) }\end{array}$ & $"$ & 100 & - & & 32 & - & $5^{\prime} 0$ & $4^{\prime} 0$ \\
\hline 13 & $\begin{array}{l}\text { Laupheim } \\
\text { (3 Proben) }\end{array}$ & " & 100 & - & & '5 & - & $3 ’ 5$ & $3^{\prime} 0$ \\
\hline
\end{tabular}

$\mathrm{KA}=$ Kalke und Dolomite der Alpen.

Alb. = Albmaterial und tertiäre Albüberdeckung (Bussen).

Gn. = alle zentralalpinen kristallinen Schiefer, Granite usw. außer Amphibolite. Am. = Amphibolite.

Schw. = rötliche kristalline Schiefer, Granite usw. des Schwarzwalds.

$\mathrm{Qu} .=$ Quarze und Hornsteine.

Bei Beachtung der geröllpetrographischen Fazien kann die Geschichte der Donau während des weitesten Gletschervorstoßes recht gut erkannt werden. Sehr regelmäßig sind folgende Fazien zu beobachten (siehe Tabelle 1, Nr. 4-6): Die B a s is fazies (6) ist ähnlich der der Jungrißschotter (1). Sie ist charakterisiert durch das Überwiegen des Materials aus dem Schichtstufenland und dem Schwarzwald mit außerordentlicher Abplattung des ersteren und guter Abplattung und Abrundung des letzteren. Das starke Hervortreten des Schwarzwaldgerölls kann nur durch kaltklimatische Wirkungen - das Herabwandern der Frostschuttzone und einer Vergletscherung des Schwarzwaldes - verstanden werden. Interessant sind in diesem Zusammenhang die jüngsten Untersuchungen über die Vergletscherung des Harzes durch Poser \& Hövermann (1951), nach denen auch hier die Vergletscherung vor dem Eintreffen des Inlandeises ihr größtes Ausmaß erreicht haben soll. 
Das hochrheinische Material der Basisfazies scheint ganz vorwiegend aus älteren Rheingletscherschottern und -moränen umgelagert worden zu sein, was sich vor allem im höheren Anteil von Restschottern alpinen Ursprungs (Kieselkalken, Hornsteinen und Amphiboliten) zeigt. Die Basisfazies entspricht also der lokalen glazialen Ablagerung im Donaugebiet bis zur Illermündung in jener Zeit des Gletschervorrückens, in der die Schmelzwässer noch nirgends die europäische Wasserscheide überfließen konnten, in der sie vielmehr restlos zum Rhein gelenkt waren.

Den Schotter, welcher in Ober-Marchtal wie aber auch an vielen anderen Stellen über der Basisfazies folgt und am mächtigsten entwickelt ist, kann man genetisch als die Vorstoßschotterfazies oder nach der Herkunft als die hochrheinische Fazies ansprechen (5). Als dritte kann man die Hang e n d fazies (4) nennen, die wieder ein Überwiegen des lokalen Materials zeigt. Dieses ist aber wesentlich schlechter gerollt und abgeplattet als im Liegenden, und die Schwarzwaldgerölle sind ganz selten geworden. Es kann kein Zweifel bestehen, daß diese Ablagerung in erster Linie bestimmt wurde durch die periglaziären Vorgänge im unmittelbar benachbarten gletscherfreien Gebiet (Bodenfließen, Aufschotterung in den Albtälern), während die akkumulativen Fernwirkungen des Rheingletschers bereits stark nachgelassen hatten. Es ist sehr interessant, daß die Hangendfazies in der letzten Donauablagerung der Albtalung nicht mehr gefunden wird, sondern nur längs dem neuen Donaulauf am Albrand. Sie ist hier in die hochrheinische Fazies diskordant eingelagert und besitzt ein tieferes Terrassenniveau als diese. Die Verlegung der Donau war also gegen das Ende des Hochglazials erfolgt.

Es muß gesagt werden, daß die genaue Datierung der unter 3.) genannten Schottervorkommen noch nicht abgeschlossen ist. Einzelne Vorkommen, wie z. B. das von Herbertingen, können auch jüngeren Stadien der großen mittelrißzeitlichen Vereisung (vor allem dem Heiligkreuztaler Stand) angehören. Aber dem Jungriß können sie nicht zugerechnet werden, da die jungrißzeitliche Vereisung sicher nicht mehr ins Rięlinger Becken gereicht hatte.

4.) Der Geschiebemergel im Hangenden des Riedlinger Schotters (5). Vielleicht gehören hierher auch andere Geschiebemergel, so die liegenden in Neufra und in Ertingen.

5.) Das Schotterfeld westlich und nördlich von Riedlingen, dessen bereits denudierte Oberfläche in der großen Kiesgrube nördl. der Stadt rund $20 \mathrm{~m}$ und dessen Sohle ca. $7 \mathrm{~m}$ über dem Donautalboden gelegen ist. An der Ostseite des Aufschlußes war im Mai 1951 über dem Schotter ein unverwitterter Geschiebemergel (4) zu sehen, darüber ein fast geröllfreier Lehm. Eine Fläche, welche Schotter und Lehmeinfüllung schneidet, ist deutlich verwittert. Über dieser Verwitterung, welche im Schotter der N- und W-Wand nur noch in einzelnen Zapfen erhalten ist, liegt ein jüngerer mächtiger Geschiebemergel (zu 2). Es ist unwahrscheinlich, daß der Liegendmergel nicht in situ gelegen, also verflossen ist. Der Schotter von Riedlingen ist demnach zweimal vom Gletscher überfahren worden; zwischen beiden Vorstößen ist eine warmklimatische Periode anzunehmen. Der Schotter ist genau so wie der des jüngeren, wahrscheinlich weitesten Vorstoßes deutlich in zwei Fazieslagen gegliedert (siehe Tabelle 1, Nr. 7 und 8). Auch hier ist die B a s is fazies der glazialen Donau sehr gut von der $\mathrm{h}$ o $\mathrm{ch}$ r hein is chen Fazies des eigentlichen Vorstoßschotters zu unterscheiden. Zu Nr. 4 der Schichtfolge gehört vielleicht auch die Bechinger Endmoräne (Blockpackung aus hauptsächlich örtlichem Material), über deren Verwitterung (!) ebenfalls noch ein frischer Geschiebemergel (2) erhalten ist. 
6.) Die Schotterakkumulation von Erting en, die nordwärts über Neufra hinaus auf einem hohen Tertiärsockel (rund $20 \mathrm{~m}$ über Talboden) den östlichen Beckenrand bildet. Die Oberfläche der Schotter liegt, je nach der Wiederausräumung durch den Gletscher, 29-36 m über Talboden, sie sinđ immer von Geschiebemergel, in Ertingen sogar von zwei Grundmoränen überdeckt, von denen die liegende noch deutlich eine Verwitterung zeigt. Die Altersstellung der Geschiebemergel ist noch nicht klar, da sie jeweils einen Gletschervorstoß älter sein können als jene über dem Riedlinger Schotter. Der Schotterkörper von Neufra zeigt schwache Stauchungen und einen mehrmaligen Fazieswechsel (siehe die Schotteranalysen 9-11 in Tabelle 1). Unmittelbar über dem Tertiär setzt die hochrheinische Fazies ein (11), darüber folgt eine stark lokal gefärbte mit wenig Schwarzwaldmaterial, aber allem Anschein nach mit umso mehr umgelagertem rheinischem Material, darüber wieder ein typisch rheinischer Vorstoßschotter mit allerdings ebenfalls viel umgelagertem Geröll (beachte den hohen Anteil der Amphibolite). Dieser Wechsel kann nur durch eine stärkere Gletscheroszillation erklärt werden, das heißt durch eine zweimalige rheinische Schüttung über die im S gelegene europäische Wasserscheide hinweg, unterbrochen von einer immer noch stark glazial betonten Donauschüttung.

7.) Schließlich ist noch ein wesentlich höher gelegener Schotterstrang ostwärts an den vorigen anschließend zu beobachten, dessen Niveau am B in s e ns tock (ostwärts Ertingen) unter Geschiebemergel bei $585 \mathrm{~m}$ und dessen Sohle bei $568 \mathrm{~m}$, also $33 \mathrm{~m}$ über Donautalboden, am Hang ausstreicht. Der Schotter ist im Gegensatz zu allen bisherigen stark verfestigt und zeigt nur die hochrheinische Fazies, ist also hier die Auffüllung einer alten Schwarzachrinne.

\section{Zur Stratigraphie im RiBtal}

Im Rißtal, der Hauptabflußrinne der sogenannten Schussenzunge des alten Rheingletschers sind folgende wichtigere Ablagerungen des Mittelpleistozäns żu beobachten:

1.) Der Dona ustetten er Terrassenschotter, der von der Iller-Mündung an das Rißtal entlang bis Barabein, nordöstl. von Warthausen, erhalten ist. Der Schotter zeigt, wie alle anderen der Rißrinne, die hochrheinische Mischung, ist bis $2,5 \mathrm{~m}$ intensiv verwittert und besitzt südl. Dellmensingen eine im Mittel 1-2 m mächtige Lehmdecke. Es handelt sich um A. PEnck's Hochterrassenschotter und um Weidenbach's (1937), bzw. Schaefer's (1940) R II. Was alle bisherigen Bearbeiter nicht erkannt haben, ist die Tatsache, daß sich dieser Schotter weder in den Komplex der Äußeren (WeIDENBACH's R I) noch den der Inneren Altendmoränen (WEIDENBACH's R II) fortsetzt, sondern daß er durch das Warthauser Engtal der Riß hindurchzieht und erst weiter beckeneinwärts mit noch jüngeren glazialen Ablagerungen verknüpft werden kann. Die Kirche von Warthausen steht auf einem Rest dieser Aufschüttungsfläche. Die Fortsetzung nach Süden ist wahrscheinlich über den Galgenberg von Biberach (564 m) und über das Hochfeld $(571 \mathrm{~m}) \mathrm{zu}$ dem flachen Endmoränenwall von Rißegg anzunehmen. Zwischen dem Rindenmooser Schlierbachgraben und dem Rißtal sind die rißzeitlichen Bildungen nicht mehr vom Gletscher überfahren worden, sie müssen also einer weiter beckeneinwärts gelegenen jüngeren glazialen Serie angehören. Sie werden als jungrißzeitlich aufgefaßt und sind korrelat der Altheimer Akkumulation, die im Donautal zwischen Ulm und Sigmaringen die 12-14 m Terrasse bildet.

2.) Das W ibling e r Terrassenniveau, das um $8-9 \mathrm{~m}$ höher liegt als das Donaustettener, kann nur bis ostwärts Gögglingen, also nur im Donautal, beobachtet werden, während es sich im Rißtal nirgends mehr findet. Außerdem 
zeigt es im Gegensatz zum Komplex von Unterweiler (3) eine Beimischung von donauglazialem Material. Es wird daher angenommen, daß es sich bei ihm um eine auf das Donautal beschränkte Bildung handelt. Am wahrscheinlichsten ist, daß es jenen Ablagerungen korrelat ist, welche weiter talauf nach der Donauverlegung bei Unter-Marchtal und nach der dadurch bedingten Tiefenerosion im Abschnitt Ulm-Zwiefaltendorf akkumuliert worden sind.

3.) Eine allgemeine Bedeutung hat dagegen der Unterweiler Schotter. Südlich Wiblingen liegt seine Oberfläche nur $4 \mathrm{~m}$ über dem Wiblinger, seine Mächtigkeit beträgt hier mindestens noch $12 \mathrm{~m}$. Man kann ihn, meist mit einer deutlichen Lehmbedeckung, von der Flur „Beim Stein“ von Unter-Kirchberg (Blatt Ulm SW) verfolgen über die „Hartäcker" von Unterweiler, die „Greutäcker" und „Fuchsäcker“ ostwärts Gögglingen, „Berg“ bei Donaustetten, „Steinhau, und „Hungerberg“ ostwärts Dellmensingen bis zu einer großen Kiesgrube am Humlanger Weg (Blatt Laupheim). Hier liegt die Oberfläche bei $506 \mathrm{~m}$, die Geröllbasis bei $492 \mathrm{~m}$ oder noch ein wenig tiefer. Sie ist also höchstens $10 \mathrm{~m}$ über dem Donautalboden und einige Meter unter dem Niveau der Donaustettener Jungrißterrasse gelegen. Die Verwitterung erreicht im Donautalabschnitt $3-4 \mathrm{~m}$. Ins Rißtal setzt sich diese Akkumulation nun nicht in den mächtigen Laupheimer Schotter fort, wie man vielleicht bei einer ersten Übersicht meinen könnte, sondern sie liegt tiefer. Da der Schotter auch im Donautal südlich Ulm sozusagen kein Albmaterial führt, muß die Donau damals noch in der Alb geflossen sein. Die Datierung des Schotters ist damit ziemlich genau gegeben: er entspricht der letzten Rheingletschermaterial führenden Aufschüttung der Alb-Donau.

Diese Altersstellung wird durch die Verhältnisse rißtalaufwärts durchaus bestätigt. Zwischen dem ununterbrochen bis Barabein zu verfolgenden Jungrißschotter und den älteren Laupheimer „Hochterrassen“ ist der nördlich Läupheim aussetzende Unterweiler Schotter wiederzufinden bei Baltringen und Äpfingen. Ér baut hier eine deutliche Terrasse auf, deren Niveau rund $10 \mathrm{~m}$ über der Jung-

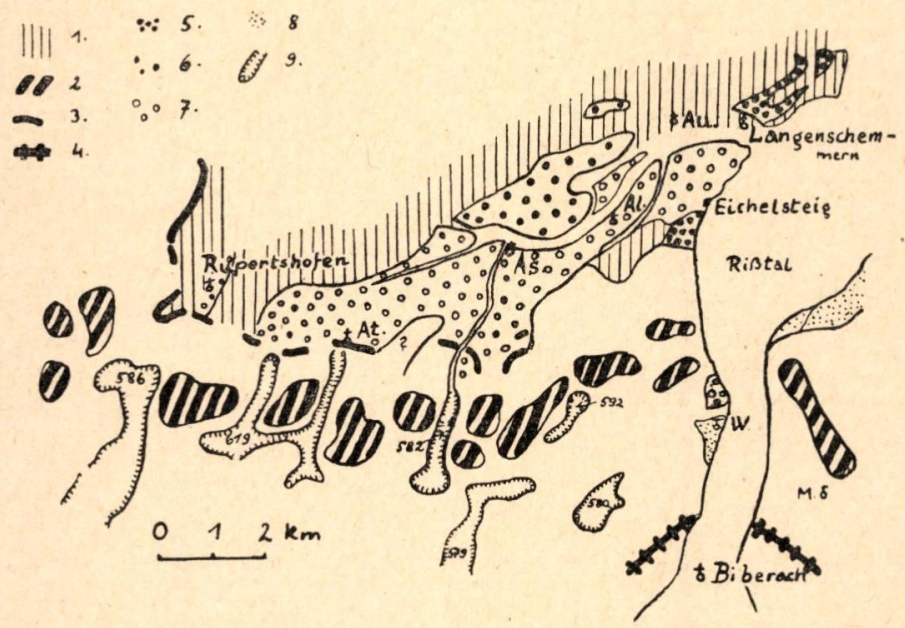

Abb. 3. Die mittelpleistozänen Ablagerungen nw. Biberach. $1=$ Tertiär, $2=$ Kuppen der Altriß-Endmoränen, 3 = Endmoräne des weitesten Rißvorstoßes (ZwiefaltendorfAttenweiler), $4=$ Biberacher Mittelriß-Endmoräne (Lindele), $5=$ Altrißschotter, $6=$ die höheren Mittelrißschotter des Aßmannharter Tales, $7=$ die tieferen Mittelrißschotter des Aßmannharter Tales (weitester Vorstoß), $8=$ Jungrißschotter, $9=$ glazigene Wannen mittelrißzeitlichen Alters. At. = Attenweiler, As. = Aßmannshart, Al. = Alberweiler, Au. = Aufhofen, W. = Warthausen, M. = Mettenberg. 
rißterrasse liegt. Sehr schön ist die gleiche Oberfläche zwischen Eichelsteig und Langenschemmern auf der anderen Talseite zu beobachten, wo auch die Schotterunterkante durch einen starken Quellhorizont angezeigt wird. Sie erreicht wenig nördlich Eichelsteig ihren tiefsten Punkt bei $521 \mathrm{~m}$ ( $3 \mathrm{~m}$ über Rißtalboden) und steigt gegen Langenschemmern wieder an. Es ist hier durch die spätere Rißerosion ein älteres Querprofil des Aßmannharter Tales angeschnitten. So erstrecken sich denn auch die Eichelsteiger Schotter das Aßmannharter Tal aufwärts bis fast nach Rupertshofen und nach Süden bis an den Rand der Altmoräne (siehe Abb. 3). Hier wurde bis jetzt angenommen, daß die Schotteroberfläche in eine genetisch einheitliche Atmoränenlandschaft übergeht.

Bei Eichelsteig hingegen liegen die Schotter völlig diskordant neben den im S auf hohem Tertiärsockel ausstreichenden Altmoränen und deren Übergangskegel nördlich Röhrwangen. Die Erklärung war erst gefunden, als ich auch südlich Aßmannshart und bei Attenweiler eine sehr interessante Diskordanz der Formengruppen festgestellt hatte. Die Eichelsteiger Schotter verknüpfen sich nur mit einzelnen kleinkuppigen Endmoränen (so im „Brandhölzle“ und bei „Baaräcker" westl. Attenweiler, im nördlichen Teil des Ortes und auf P. 600'7 am Gänsberg - alles auf Bl. Warthausen). Mit den um 25 bis $45 \mathrm{~m}$ höheren und mächtigen Endmoränen des Hauptwalles aber haben sie nichts zu tun. Zum Eichelsteiger Schotter gehören als Formelement ferner die eigenartigen wannenförmigen Durchbrüche durch den genannten hohen Hauptmoränenwall: das Schammacher Tal, das Gutershofener Tal und die Wanne des Langen Weihers bei Burren. Auch im Burrenwald quert noch eine solche Furchung den Hauptwall. Südlich derselben liegen kleine, fast abflußlose Becken: Neuer Weiher, Alter Weiher, Oberer Weiher und südlich von Burren, die ebenfalls $\mathrm{zu}$ dieser Formengruppe gehören (siehe Abb. 3). Es ist mir höchst wahrscheinlich, daß alle diese eigenartigen Hohlformen glazigener Entstehung sind und daß sie einem Gletscher entsprechen, der sich längere Zeit am Hauptwall gestaut hat, diesen nachher zwar nicht überfahren, aber an mehreren Stellen durchbrechen konnte. Die flachen Endmoränen nördlich des Hauptwalles zeigen den äußersten Stand jenes Gletschers an, die Eichelsteiger Schotter sind die Ablagerungen seiner Schmelzwässer. Es ist zu ergänzen, daß zumindest die Hohlformen bei Burren (Langer Weiher) nicht von einem einzigen Gletscherdurchbruch durch den Hauptendmoränenwall entstanden sein können, da sich nördlich Aßmannshart mit etwas höherem Niveau und höherer Unterkante noch ein zweiter glazialer Schotterhorizont findet, der ebenfalls nur durch die Furche des Langen Weihers hertransportiert worden sein kann. Es ist daher nicht verwunderlich, wenn die Hohlform des Langen Weihers eine deutlichere Gletschererosion zeigt als die anderen Durchbrüche.

Im Biberacher Rißengtal treten die korrelaten Schichten erst bei Schloß Warthausen auf. Wandert man über die Höhe, wird einem die $\mathrm{m}$ or $\mathrm{ph}$ olog is $\mathrm{ch}$ e Diskordanz klar, die zwischen der ausgesprochenen Ebenheit der Schotterterrasse und dem im Westen anschließenden kuppigen Moränengelände besteht. Im Birkenharder Tälchen aber kann man ohne weiteres die g e olog is ch e Diskordanz feststellen zwischen den bis zur Talsohle reichenden Schottern im Osten und dem auf höherem Tertiärsockel (Obere Meeresmolasse aufgeschlossen) ruhenden älteren Glazialkomplex im Westen. Die mächtigen Schotter, auf denen das Warthauser Schloß steht, sind also an ältere glaziale Schichten angelagert. Rißtalaufwärts ist ihre Fortsetzung in den riesigen Aufschlüssen nördlich der Stadt Biberach zu finden. Wie schon Krauss (1932) und Weidenbach (1937) beschrieben haben, liegen hier über mächtigen glazialen Schottern zuerst ein Geschiebemergelband $(3-4 \mathrm{~m})$, dann abermals Schotter, die sich talauf deutlich mit 
Endmoräne verzahnen. Es sind dies die Endmoränen vom Lindele (bei WEIDENBACH R II), deren Bogen sich über Mittelbiberach einerseits, und über Bergerhausen und den Jordanberg andererseits erstreckt. Dies ist einer der deutlicheren Wälle in $n$ e $r$ halb des älteren Doppelwalles von Warthausen. Er kann natürlich nicht gleichalt sein mit den oben geschilderten Endmoränen von Attenweiler usw. Das zwischen Biberach, Mettenberg und Bergerhausen verbreitete Geschiebemergelband im Liegenden und außerhalb der Lindele-Moräne spricht dafür, daß diese jünger ist, das Geschiebemergelband aber einem äuß e ren Stand entsprechen könnte. Jedenfalls gehört die Attenweiler Endmoräne zu dem äußersten Stand von Zwiefaltendorf im Riedlinger Becken, da sie, nur mit kleinen Unterbrechungen, von der Kuppe 618'0 südl. Rupertshofen, südlich und westlich um die Buchhalde, über das „Himmelreich", den Sonnenberg, das Ziegelgehau bei Saugart, ostwärts Klingen, das Klingengehau, P. 550'4 bei Köhlberg, den „Tiergarten“, „Heilenspitz“ und den Rücken südlich Oberwachingen bis nördlich Offingen, südlich und westlich um den Bussen zum P. 596'0 westlich Dietelhofen, über Hühnerbühl und das Schlatthau zum Zwiefaltendorfer Wall zu verfolgen ist (die angegebenen Örtlichkeiten sind alle auf dem Blatt Uttenweiler der Württemberg. Topogr. Karte 1:25000 $\mathrm{zu}$ finden).

4.) Die Schotter der stark verlößten hohen Terrasse von $\mathrm{L}$ a u p h e i m, die bei Penck \& Brückner noch zu Mindel gestellt worden waren (seit Weidenbach aber ins Riß I), stammen zum allergrößten Teil aus den östlichen Zuflüssen der Riß. Erst halbwegs zwischen Baustetten und Laupheim, wo die Geröllunterkante auffallend schnell absinkt, weshalb hier auch an eine Anlagerung verschieden alter Akkumulationen gedacht werden kann, zeigt sich die kristallinreichere $\mathrm{Zu}$ sammensetzung, die für die Rißrinne typisch ist. Die entsprechenden Ablagerungen sowohl im Rottum- als auch im Rottal sind wesentlich ärmer an zentralalpinen Bestandteilen (siehe Tabelle 1, Nr. 12-13). In der Stadt Laupheim liegt die Unterkante des Schotters wenig über der Oberfläche der benachbarten Jungrißterrasse oder $13 \mathrm{~m}$ über dem Rißtalried. Dieser bei Laupheim noch über $15 \mathrm{~m}$ mächtige Schotterzug kann talab nur bis zur Flur „Regel“ nordöstlich Stetten verfolgt werden. Talauf sind Äquivalente sehr wahrscheinlich südlich Äpfingen zu finden. Wie die Verbindung mit den Warthauser Altrißmoränen erreicht werden kann, ist aber noch unklar. Der Übergangskegel nördlich von Röhrwangen liegt jedenfalls zu hoch.

\section{Zur Stratigraphie im Rottal}

Die östlichste Schmelzwasserrinne des Rheingletschers lag im Bereich der Württembergischen Rot. Sie ist sicher schon im Altdiluvium vorhanden gewesen, wofür die hier noch gut erhaltenen „Unteren und Oberen Deckenschotter" sprechen. Jünger als sie sind eine Reihe von ebenfalls gut erhaltenen, aber ausgesprochen rinnenartigen Schotterauffüllungen glazialer Natur. Der stratigraphische Vergleich zeigt, daß diese zum allergrößten Teil älter sein müssen als jene Ablagerungen, welche im Donau - Bussen - Federsee - Gebiet dem weitesten Stand des Mittelriß angehören. Es sind im Rottal folgende verbreitetere Schichtglieder festzustellen:

1.) Die Donaustettener Akkumulation läßt sich im Rottal nur bis zum Orte Bronnen (Oberfläche bei $509 / 10 \mathrm{~m}$ ) verfolgen. Durch einzelne kleine Terrassen, auf denen meist Ortschaften gelegen sind, werden die nur wenige Meter mächtigen Schotter dieser Ablagerungszeit verraten, so in Hochstetten bei $518 / 19 \mathrm{~m}$, in Orsenhausen, nördlich Schwendi in 536 und $538 \mathrm{~m}$, deutlicher bei Bechtenrot 
in $572 / 3 \mathrm{~m}$, immer noch $8-9 \mathrm{~m}$ über dem heutigen Rottalboden. Hier sind auch die Schotter aufgeschlossen. Sie zeigten die typische, völlig entkalkte und ausgelesene Fazies der Restschotter, also einer periglaziären fluviatilen Ablagerung, wie man sie in allen autochthonen Tälern der Schotterriedelzone des Alpenvorlandes antrifft (vgl. H. Graul 1937, 1943). Talauf finden sich weitere Reste dieser Verschotterung.

Tabelle 2

\begin{tabular}{|c|c|c|c|c|c|c|c|}
\hline $\mathrm{Nr}$. & Ort: & Alter: & KA. & Gn. & $\mathrm{Qu}$. & Harte. & Sdst. \\
\hline 1 & $\begin{array}{l}\text { Haderhöhe bei } \\
\text { Orsenhausen } \\
\text { (554 m üb. N.N.) }\end{array}$ & Mindel & 100 & $2^{\prime} 0$ & 0 '6 & $1 ' 3$ & $1 ' 7$ \\
\hline 2 & $\begin{array}{l}\text { Gem. Kgr. Eichen } \\
(570 \mathrm{~m})\end{array}$ & Altri $B$ & 100 & 377 & 0 '8 & $1 ' 3$ & 2'8 \\
\hline 3 & $\begin{array}{l}\text { Gem. Kgr. Gutenzell } \\
(574 \mathrm{~m})\end{array}$ & " & 100 & $8 ’ 0$ & $1 ’ 7$ & $2 ’ 5$ & $1 ’ 0$ \\
\hline 4 & $\begin{array}{l}\text { Gem. Kgr. Edel- } \\
\text { beuren }(583 \mathrm{~m})\end{array}$ & $"$ & 100 & $14^{\prime} 0$ & $0 ’ 5$ & $0 ’ 9$ & - \\
\hline 5 & $\begin{array}{l}\text { Gegenüber Edel- } \\
\text { beuren }(573 \mathrm{~m})\end{array}$ & Mittelriß & 100 & $51^{\prime} 5$ & $4^{\prime} 3$ & $12 ' 8$ & $4 ’ 5$ \\
\hline 6 & Erolzheim $(545 \mathrm{~m})$ & Würm & 100 & $6^{\prime} 4$ & $3{ }^{\prime} 0$ & $1 ' 0$ & $1^{\prime} 0$ \\
\hline
\end{tabular}

$\mathrm{KA}=$ wie Tabelle 1 .

$\mathrm{Gn} .=$ alle zentralalpinen kristallinen Schiefer, Granite usw.

Qu. = nur Quarze.

Harte = Quarzite und Hornsteine, auch sehr harte Kieselkalke.

Sdst. $=$ Sandsteine.

Die Geröllanalysen wurden an der frischen Schotterwand an den Korngrößen von rund $25-75 \mathrm{~mm}$ durchgeführt.

2.) Auch eine ältere Einlagerung ist kaum besser erhalten. Es handelt sich um die etwa $10 \mathrm{~m}$ mächtige Geröllablagerung des Ulmer Gehau. Sie ist zum Teil, und zwar die liegende Hälfte, glazigener Entstehung. Ihren Ausgang nahm sie von der Endmoräne bei Ellwangen (Blatt Wurzach), wie SchreINER (1950) nachweisen konnte. Von Ellwangen ist diese Schotterterrasse fast ununterbrochen bis gegen Spindelwag im Ölbachtal erhalten. Sie ist nach Höhenlage der Aufschüttungssohle, des Niveaus und nach der petrographischen Zusammensetzung ohne weiteres mit dem Terrassenschotter des Ulmer Gehaus zu verknüpfen. Talab kommt dieser in der Umgebung von Edelbeuren vor und ist vor allem gegenüber dem Orte gut aufgeschlossen. Die Geröllanalyse zeigt einen hohen Anteil an Amphiboliten und anderer harter Restgesteine (siehe Tabelle 2, Nr. 5). A. Schreiner bezeichnete die ganze Serie als Jungriß, da er die Rißablagerungen nur in zwei Gruppen gliederte. Der Ellwangener Stand ist aber unzweifelhaft älter als die dem Donaustettener Jungriß korrelaten periglaziären Bildungen des Rottales; er ist bestimmt dem Mittelriß zuzurechnen und höchstwahrscheinlich dem weitesten Stand zwischen Donau und Rißtal, nämlich dem von Zwiefaltendorf-Attenweiler. Auch im Gebiet des Wurzacher Teilzungenbeckens dürfte sich nachweisen lassen, daß dieser Gletscherstand über die Ausdehnung älterer mittelrißzeitlicher Stände hinausgegangen ist.

3.) Älter als der ganze mittelrißzeitliche Komplex sind alle jene Schotter, die sich von Laupheim das Rottal aufwärts über Schwendi, Gutenzell, Mettenberg und Haslach als der kristallinärmere $\mathrm{Haslach-Gutenzeller} \mathrm{Zug} \mathrm{einer-}$ seits und über Weitenbühl, Eichenberg nach $\mathrm{Th}$ a $\mathrm{n} \mathrm{h}$ ei $\mathrm{m}$ als ein wesentlich 
kristallinreicherer Schotterstrang andererseits fortsetzten. K. ScHÄDEL (1950) hat sie petrographisch unterschieden und nur den kristallinreicheren Strang - trotz seiner höheren Auflagerungsfläche im mittleren Rottal - in die Rißzeit, den kristallinärmeren Haslacher aber ins Mindel (II) gestellt. Ich kann mich dieser Ansicht nicht voll anschließen, obwohl die Verhältnisse auf den Blättern Leutkirch und Aitrach zum Teil für ScHäDEL sprechen. Die Lageverhältnisse der Ablagerungen im nördlichen Terrassengebiet lassen vorläufig keine andere Deutung $\mathrm{zu}$, als daß 1.) sowohl der Haslach-Gutenzeller als auch der Thannheimer Schotter rißzeitlich sind und 2.) der letztere älter ist als der erstere. Der Komplex des relativ kristallinarmen und mächtigen Schotters liegt sowohl im unteren Rottal als auch im Rottumtal sehr tief. In letzteres wurde zwischen der Platte des Oberen Deckenschotters von Erlenmoos im Süden und den mit Resten des Unteren Deckenschotters bedeckten Kuppen des Sandberges bei Schönebürg und der Walpertshofener Höhe (Blatt Schwendi der Karte 1:25000) ein Schotterstrang abgelagert. Der Höhenunterschied seiner Basis zum Unteren Deckenschotter beträgt im Rottal bis zu $25 \mathrm{~m}$, im Rottumtal sogar bis $40 \mathrm{~m}$. Dazu kommt, daß die Unteren Deckenschotter einwandfrei mit noch mächtig erhaltenem Verwitterungshorizont unter die gesamte Altmoränenlandschaft einstreichen, die Laupheim-Gutenzell-Haslacher Schotter aber in die älteren Teile der Altmoränen eingelagert erscheinen. Es besteht demnach eine der sichersten stratigraphischen Grenzen zwischen dem mächtig verwitterten Unteren Deckenschottern einerseits und den rinnenartigen Schotterkomplexen wie den Altmoränen andererseits, deren Herausarbeitung wir ja WEIDENBACH (1937) verdanken (siehe Abb. 1).

Die Tabelle 2 läßt den Unterschied im zentralalpinen Geröllanteil bei den einzelnen Schotterkörpern gut erkennen. Während beim altpleistozänen Schotter der Holzstöcke (1) nur 2 Kristallingerölle auf 100 Kalke kommen, enthält der Laupheimer (2) in der Regel $3^{\prime} 5-5^{\prime} 5$. Dieser immer noch geringe Kristallingehalt entspricht ganz jenem, den wir bei den Rinnenfüllungen antreffen, die im östlichen Grenzgebiet zwischen Rhein- und Illergletscher Material von beiden erhalten haben. Siehe dazu den Kristallin-Hundertsatz z. B. der würmzeitlichen Schotter im heutigen Illertal (6). In den hangenden Partien oder, was weit wahrscheinlicher ist, in einer älteren, um etwa $10 \mathrm{~m}$ höher gelegenen Aufschüttung, die vom Rücken ostwärts Schwendi über die Gutenzeller Gemeindekiesgrube talauf zu verfolgen ist (3), erreicht der Kristallingehalt 8-9 auf 100 Kalke. Der Weitenbühl-Eichenberg-Thannheimer Schotter (4) zeigt aber von der Basis an 14-18 Teile Zentralalpin. Und die eingelagerten mittelrißzeitlichen, weitgehend umgelagerten (sekundären) Schotter (5) haben einen noch wesentlich höheren Anteil, von dem die Hälfte widerstandsfähige Amphibolite sind. Es kann keinem Zweifel unterliegen, daß wir in der Rotrinne mehrere Schotterstränge sowohl schotteranalytisch als auch nach der Höhenlage der Aufschüttungssohle unter-

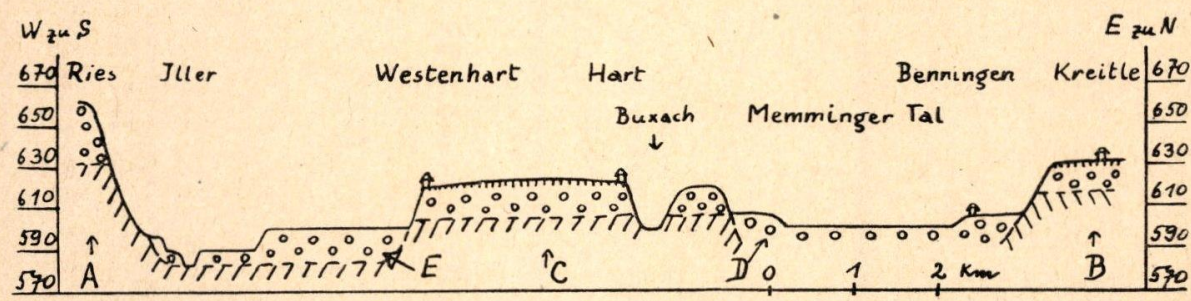

Abb. 4. Querprofil Illertal - Memminger Tal. A = Thannheimer Schotter (Altriß), $\mathrm{B}=$ Hawangener Feld (älteres Mittelriß), $\mathrm{C}=$ Hitzenhofener Feld (jüngeres Mittelriß), $\mathrm{D}=$ würmzeitliche Verschotterung im Memminger Tal, $\mathrm{E}$ = desgleichen im Illertal. 
scheiden können, die alle älter sind als der Zwiefaltendorf-Attenweiler-Ellwangener Gletscherstand (Mittelriß). Andererseits aber sind jene Schmelzwasserablagerungen jünger als die mächtige fossile Verwitterungsrinde der Heggbacher Unteren Deckenschotter. Ich stelle jene Schotterkomplexe ins A ltri $B$, da ich vorläufig keine Veranlassung sehe, P. BEck's (1933) Beispiel zu folgen und hier eine eigene Vereisungsgruppe abzusondern. Das stratigraphische Verhältnis zu den zwei Hauptendmoränenwällen der Altriß ist noch zu klären.

Bei einem Vergleich mit den Ablagerungen im benachbarten Illergebiet fällt auf, daß die beiden gut erhaltenen Schotterhorizonte bei Memmingen - das Hitze $n$ h of e n e r und das $\mathrm{H}$ a w a $\mathrm{n}$ g e $\mathrm{n}$ e r F eld - die von I. Schaefer(1940) als Riß I und II bezeichnet worden waren, jünger sein müssen als die Altrißablagerungen der Rotrinne und älter als die jüngsten rißzeitlichen Ablagerungen des Riß- und des Donautales. Sie sind also Mittelriß. Aus dieser Erkenntnis ergibt sich ohne weiteres die Notwendigkeit, die Altrißbildungen des Rheingletschers auch im Illergebiet $\mathrm{zu}$ verfolgen und vor allem die im Rheingebiet so deutliche Grenze zwischen den Unteren Deckenschottern und dem mittelpleistozänen Komplex auch hier genauer als bisher zu fassen.

Zusammenfassend können in den Hauptabflußrinnen des nördlichen Rheingletschers folgende mittelpleistozäne Horizonte festgehalten werden:

\begin{tabular}{lccc}
\hline Alter: & Donautal: & Rißtal: & Rottal: \\
\hline Jungriß: & $\begin{array}{c}\text { Schotter von Altheim } \\
\text { Unlingen }\end{array}$ & $\begin{array}{c}\text { Donaustettener Schotter } \\
\text { und wahrscheinlich Riß- } \\
\text { egger Endmoräne }\end{array}$ & $\begin{array}{c}\text { periglaziäre } \\
\text { Terrassenschotter }\end{array}$
\end{tabular}

Innere Moränenwälle, so z. B. Heiligkreuztal mit Schotterfeld von Andelfingen, event. auch Herbertinger Terrassenschotter.

Mittelriß: $\quad$ Zwiefaltendorfer Endmoräne (weitester Stand) mit Marchtaler Schotterfeld, letzte Donaufüllung in der Kirchener-

Schmiech-Blau-Talung.

Riedlinger Schotter mit der ihn überlagernden Liegend-Grundmoräne.

Schotter von Ertingen Neufra

Altriß: Schotter vom Binsenstock

Wahrscheinlich die hohen Mindel: $\quad$ Schotter von Emeringen.

Innere Moränenwälle, z. B. Lindele, Gigele etc. bei Biberach, Wiblinger Schotterterrasse.

Attenweiler Endmoräne mit Schotter des ABmannharter Tales (Eichelsteig); wahrscheinlich das Geschiebemergelband im Warthauser Rißtal; Unterweiler Terrassenschotter.

Höherer Schotterhorizont des Aßmannharter Tales.

Laupheimer Schotterterrasse; Warthauser Endmoränenwälle verschiedener Stadien.

"

Liegendschotter von Winterstetten-Reichenbach, Teile vom Hochgelände usw. periglaziäre Bildungen.

Ellwanger Endmoräne mit Schotter des Ulmer Gehau. ?

LaupheimSchwendiGutenzellHaslacher Komplex. EichenbergThannheimer Schotter.

Unterer Deckenschotter von Heggbach und den Holzstöcken. 
Vergegenwärtigen wir uns, daß man im nördlichen Alpenvorland für die letzte Eiszeit allenthalben bereit ist, zwei Stadien anzunehmen (ScHAEFER 1940, RATHJENs 1951), für die beiden daraus zu folgernden Akkumulationsphasen aber im ganzen Donautal, im Rhein-, Lech-, Inn- und Salzachgletschergebiet keine zwei selbständige Schotterkörper finden konnte, dann muß einem die äußerst reichhaltige Stratigraphie des Mittelpleistozäns im westlichen deutschen Alpenvorland zu denken geben. Die Schichtglieder füllen die lange und wirkungsvolle Erosionszeit zwischen dem Niveau der Unteren Deckenschotter und der Basis der letzteiszeitlichen. Schotterfelder aus und zwar in der Weise, daß bereits vor dem verhältnismäßig jungen äußersten Stand des westlichen Rheingletschers (dem jüngeren Mittelriß) der größte Teil der Tiefenerosion erfolgt war. Diese Verhältnisse erinnern uns sehr an die des Schweizer Vorlandes. Auch hier liegen vor dem weitesten Stand die tiefen Rinnenschotter, auch hier die deutliche Zäsur zwischen dem Unteren Deckenschotter und allem Jüngeren.

Ich habe - zum ersten $\mathrm{Mal}$ in einem Vortrag im Kolloquium des Geologischen Institutes der Universität Tübingen im Februar 1949 - vorgeschlagen, sich einer elastischeren Gliederung des Mittelpleistozäns zu bedienen und von der starren Numerierung einzelner Schichtglieder mit I-III abzukommen, da in den verschiedenen Schmelzwasserrinnen ganz verschiedene Glieder erhalten sind, die man bei einer großmaßstabigen Kartierung unmöglich miteinander altersgleich setzen kann. Ich schlug die Gliederung in Alt-, Mittel- und Jungriß vor und glaube, daß sich diese Einteilung inzwischen bereits recht gut bewährt hat.

Die wichtigsten Folgerungen aus den Ergebnissen dieses ersten Berichtes sind:

1.) Die reichhaltige Stratigraphie des Mittelpleistozäns kann nur auf eine öftere Aufeinanderfolge von Kalt- und Warmphasen und der davon abhängigen geologischen, morphologischen und biologischen Verhältnisse zurückgehen. Eine allgemeine Gleichsetzung (quantitativ und qualitativ) von „Rißzeit“ und „Würmzeit" ist nicht möglich, ohne zu vollkommen verkehrten Schlüssen für die Rißzeit zu kommen. Die Rißzeit muß unverhältnismäßig länger gedauert haben als die Würmzeit. Siehe dazu auch die sprunghafte Zunahme der Verwitterungsmächtigkeit von Würm- zu Rißablagerungen.

2.) Die lange Dauer des Mittelpleistozäns, dessen Vereisungen als „Rißeiszeitengruppe" bezeichnet werden, nötigt uns, die Ursachen für petrographische und morphologische Diskrepanzen innerhalb seiner Ablagerungen auch in Faktoren zu suchen, die während der Würmzeit nicht mehr wirksam gewesen zu sein scheinen. Gedacht wird dabei insbesondere an Phasen verstärkter en bloc Hebung des Vorlandes, womit die Tiefeneinschneidung belebt und die rinnenartige Ablagerung gewisser glazigener Schotter verständlicher würde (siehe da$\mathrm{zu}$ analoge Vorstellungen Schweizer Forscher).

3.) Zur Erforschung der postrißzeitlichen Morphogenese unserer Landschaft können nur einwandfrei postjung rißzeitliche Bildungen herangezogen werden, da wir sonst nicht die Entwicklung der Postrißzeit, sondern die der Postmittelrißzeit erfassen. Diese ist aber wesentlich länger und hat einen öfteren klimatischen Wechsel mitgemacht als jene. Ebenso ist bei der Altersbestimmung von Lößprofilen auf Rißschottern in jedem Falle erst festzustellen, welcher Untergruppe diese angehören. Auf Mittelrißschottern, und diese setzen den allergrößten Teil unserer sogenannten „Hochterrassen“ im deutschen Alpenvorland zusammen, können eben auch Jungrißlöße oder nur Würmlöße oder beide liegen.

4.) Die Frage nach der alt-/mittelpleistozänen Grenze ist im bayerisch-österreichischen Vorland erneut zu überprüfen. 


\title{
Literaturverzeichnis.
}

Beck, P.: Über das schweizerische und europäische Pliozän und Pleistozän. - Eclog. geol. Helv. 1933. - Vorläufige Mitteilung über eine Revision des alpinen Quartärs. - Ebendort 1937.

Bräuhäuser, M.: Beschreibung des Rißgelbietes. - Württ. Min. d. Innern, Bericht d. Straßen- u. Wasserbauverwaltung, 1921.

Ge ologische Úbersich ts kartierung Südwürttembergs unter Leitung von G. WAGNER, 1946-49: die Blätter Saulgau (F. WENK), Munderkingen und Uttenweiler (HeinzelmanN), Schwendi, Warthausen und Laupheim (H. Graul), Aitrach (K. SchëDEL u. a.).

Graul, H.: Untersuchungen über Abtragung und Aufschüttung im Gebiet des unteren Inn und des Hausruck. - Mitt. geogr. Ges. München 1937. - Zur Morphologie der Ingolstädter Ausräumungslandschaft. - Forsch. z. deutschen Landeskunde 43, 1943.

Graul, H., Weidenbach, F. \& Schaefer, I.: Quartärgeologische Exkursion durch die RißLechplatte. - Geol. Bavar. Nr. 6, München 1951.

GrIPP, K.: Über den morphologischen Nachweis großer Schwankungen des Eisrandes. Eiszeit u. Gegenwart 1, S. 65. 1951.

Krauss, K.: Über die sogenannten Deckenschotter des Rißtals. - Jber. u. Mitt. oberrhein. geol. Ver. Bd. 21, S. 66. 1932.

Penck, A. \& BrǘCKNer, E.: Die Alpen im Eiszeitalter. 1901/09.

Poser, H. \& HövermanN, J.: Untensuchungen zur pleistozänen Harzvergletscherung. Abh. braunschw. wiss. Ges. 3, S. 61-115. 1951.

Rathjens, C.: Über die Zweiteilung der Würmeiszeit im nördlichen Alpenvorland. Pet. Mitt. 1951, S. 89-97.

SсHÄDEL, K.: Untersuchungen über das Altdiluvium des Rheingletschers zwischen Iller und Bodensee. Diss. Tübingen 1950 (Manuskript).

SchaEfER, I.: Die Würmeiszeit im Alpenvorland zwischen Riß und Günz. - Augsburg 1940. - Die diluviale Erosion und Akkumulation. - Forsch. z. deutschen Landeskunde 49, 1950.

SснміDт, M.: Geolog. Spezialkarte von Württemberg 1:25000, Blatt Sigmaringen, Stuttgart 1935.

Schreiner, A.: Die Entstehung des Wurzacher Beckens. - Diss. Tübingen 1950 (Manuskr.).

Trols, C.: Bericht über die Deuqua-Tagung 1950 in München. - Erdkunde 1951, S. 86.

WeIDENBACH, F.: Geolog. Spezialkarte v. Württemb. $1: 25000$, Blätter Ochsenhausen u. Biberach. Stuttgart 1937. - Bildungsweise und Stratigraphie der diluvialen Ablagerungen Oberschwabens. - N. Jb. f. Min. usw. Beil. Bd. 78, Abt. B. 1937. - Geologische Karte von Oberschwaben 1:100000, Blatt Ulm. - Stuttgart 1951 (im Erscheinen begriffen).

\section{Die Entstehung der Seen in den ehemals vergletscherten Gebieten}

\author{
Von Paul Woldstedt. Mit $3 \mathrm{Abb}$.
}

I.

In früheren Arbeiten $(1923,1926)$ habe ich die Ansicht vertreten, daß die Hohlformen der Rinnenseen usw. in den Randgebieten der ehemals vergletscherten Gebiete im wesentlichen durch Schmelzwasserströme unter dem Eis gebildet worden seien. Ich stützte mich dabei hauptsächlich auf Arbeiten von N. V. Ussing (1903) und E. Werth (1907, 1909, 1914). Der zuerstgenannte Autor zeigte bei der Untersuchung der großen jütischen Heideebenen, daß diese Sanderflächen der Letzten Vereisung sich aus einzelnen, deutlich individualisierten Schwemmkegeln zusammensetzen. An der Spitze dieser Kegel liegt das gröbste Material; nach auswärts nimmt die Korngröße ab. Die Lage der Kegelspitzen ist dadurch charakterisiert, daß hier die Endmoräne unterbrochen ist und an 